PROCEEDINGS OF THE

AMERICAN MATHEMATICAL SOCIETY

Volume 137, Number 2, February 2009, Pages 669-677

S 0002-9939(08)09693-7

Article electronically published on September 16, 2008

\title{
ON THE RADIUS OF ANALYTICITY OF SOLUTIONS TO THE THREE-DIMENSIONAL EULER EQUATIONS
}

\author{
IGOR KUKAVICA AND VLAD VICOL
}

(Communicated by Matthew J. Gursky)

\begin{abstract}
We address the problem of analyticity of smooth solutions $u$ of the incompressible Euler equations. If the initial datum is real-analytic, the solution remains real-analytic as long as $\int_{0}^{t}\|\nabla u(\cdot, s)\|_{L^{\infty}} d s<\infty$. Using a Gevrey-class approach we obtain lower bounds on the radius of space analyticity which depend algebraically on $\exp \int_{0}^{t}\|\nabla u(\cdot, s)\|_{L^{\infty}} d s$. In particular, we answer in the positive a question posed by Levermore and Oliver.
\end{abstract}

\section{INTRODUCTION}

The existence and uniqueness of $H^{r}$-solutions, for $r>3 / 2+1$, of the threedimensional incompressible Euler equations on a maximal time interval $[0, T)$, for some $T \in(0, \infty]$, is classical [EB, Ka, MB, T]. Beale, Kato, and Majda BKM] proved that if the maximal time of existence $T$ is finite, the vorticity $\omega$ satisfies $\int_{0}^{T}\|\omega(\cdot, t)\|_{L^{\infty}}=\infty$. In two dimensions it is well-known (cf. [J]) that $T$ can be taken arbitrarily large.

It is common to write the initial value problem associated to the Euler equations in terms of the vorticity $\omega=\operatorname{curl} u$ :

$$
\begin{aligned}
& \partial_{t} \omega+u \cdot \nabla \omega=\omega \cdot \nabla u, \\
& u=K * \omega, \\
& \omega(0)=\omega_{0}=\operatorname{curl} u_{0},
\end{aligned}
$$

where $K$ is the Biot-Savart kernel. Here we work in the periodic setting; that is, $u$ and $\omega$ are $\mathbb{T}^{3}$-periodic functions with $\int_{\mathbb{T}^{3}} u=0$, where $\mathbb{T}^{3}=[0,2 \pi]^{3}$. The case of the whole space can be treated with minor modifications.

In three dimensions, if the initial datum $\omega_{0}$ is analytic, Bardos $B$ and Benachour $\mathrm{Be}$ obtained lower bounds on the radius of analyticity of the solution that vanish in finite time. In $\mathrm{BB}$ they also proved persistency; i.e. the solution remains analytic as long as it exists in a certain Hölder-type space on the complexified domain. The proof is an implicit argument which does not yield an explicit rate of decay for the radius of analyticity of the solution. In the two-dimensional case, using the absence of the vorticity stretching term, Bardos, Benachour, and Zerner [BBZ] established an explicit bound for the rate of decay of the analyticity radius, which is $C \exp (-C \exp (C t))$, for a suitable positive constant $C$. The local propagation of

Received by the editors November 13, 2007.

2000 Mathematics Subject Classification. Primary 76B03, 35L60.

Both authors were supported in part by the NSF grant DMS-0604886. 
analyticity was considered by Baouendi and Goulaouic [BG], Alinhac and Metivier AM] and Le Bail [Lb].

Using a Fourier space method, Levermore and Oliver [LO proved analyticity for a generalized Euler equation in two dimensions. Their proof extends to higher dimensions and shows that the uniform analyticity radius of the solution decays exponentially in $\|\omega(\cdot, t)\|_{H^{r}}$, where $r$ is large enough. In two dimensions, this radius decays exponentially faster than the radius obtained by Bardos and Benachour. In [LO, Remark 4] the authors pose the question of whether the Fourier-based method can be employed to recover the 2D-rate obtained by Bardos, Benachour, and Zerner. We answer this question in the positive. Moreover, in the case of the 3D Euler equations, we obtain lower bounds on the rate of decay of the uniform space analyticity radius that depend only algebraically on $\|\omega(\cdot, t)\|_{H^{r}}$ and $\exp \left(\int_{0}^{t}\|\nabla u(\cdot, s)\|_{L^{\infty}} d s\right)$, improving previously known results. The results hold for the non-analytic Gevrey classes (cf. Remark 3.2 below).

The aforementioned Fourier space method, namely the Gevrey-class regularity, was introduced by Foias and Temam [FT] to prove the analyticity of solutions for the Navier-Stokes equations. This technique is general and has been applied to other equations [BGK1, BGK2, CTV, FTi, GK1, K1, LO, OT]. Analyticity in $L^{p}$ for the Navier-Stokes equations was established in [GK2, K2, L1, L2].

Section 2 contains the statement and the proof of our main result. The following are valid in any dimension $d \geq 2$, but we only state the results for $d=3$. The core of the proof of Theorem 2.1 is Lemma 2.5, whose proof is given in Section 3 ,

\section{The ANALYTICITY THEOREM}

The following is our main theorem.

Theorem 2.1. If $u_{0}$ is divergence-free, and $\omega_{0}=$ curl $u_{0}$ is real-analytic on $\mathbb{T}^{3}$, then the unique solution $\omega \in C\left(0, T ; H^{r}\left(\mathbb{T}^{3}\right)\right)$, with $r>7 / 2$, to the vorticity equations (1.1) -(1.3) is real-analytic for all $t<T$, where $T \in(0, \infty]$ is the maximal time of existence. Furthermore, the uniform space analyticity radius $\tau(t)$ of the solution $\omega(\cdot, t)$ satisfies

$$
\tau(t) \geq C_{1} \exp \left(-C_{2} \int_{0}^{t}\|\nabla u(\cdot, s)\|_{L^{\infty}} d s\right)\left(1+t^{2}\right)^{-1}
$$

where $C_{2}>0$ is a constant depending only on $r$, and $C_{1}>0$ has additional dependence on $\omega_{0}$ (cf. (2.6) below).

Remark 2.2. The theorem remains valid in any dimension $d \geq 2$, with the modification $r>(d+4) / 2$. This is due to the fact that for $d=2$ the term $\omega \cdot \nabla u$ vanishes, and that for $d \geq 4$ the vorticity formulation of the Euler equations is similar to (1.1) -(1.3) .

Remark 2.3. In dimension 2, we can take $T$ arbitrarily large and therefore the solutions remain analytic for all time. In this case $\|\nabla u(\cdot, t)\|_{L^{\infty}}$ increases with a rate at most $C \exp (C t)$ for some positive constant $C$, while $\|\omega(\cdot, t)\|_{H^{r}}$ increases with a rate at most $C \exp (C \exp (C t)$. This allows us to recover the $2 \mathrm{D}$-rate of decay given by Bardos, Benachour and Zerner [BB, $\mathrm{BBZ}$. 
The functional setting for the present paper is as follows. For fixed $r, \tau \geq 0$ and $m=1,2,3$, we define

$$
\begin{aligned}
D\left(\Lambda_{m}^{r} e^{\tau \Lambda_{m}}\right)=\left\{\omega \in H^{r}\left(\mathbb{T}^{3}\right)\right. & : \operatorname{div} \omega=0, \\
& \left.\left\|\Lambda_{m}^{r} e^{\tau \Lambda_{m}} \omega\right\|_{L^{2}}^{2}=(2 \pi)^{3} \sum_{k \in \mathbb{Z}^{3}}\left|k_{m}\right|^{2 r} e^{2 \tau \mid k_{m}}\left|\hat{\omega}_{k}\right|^{2}<\infty\right\},
\end{aligned}
$$

where

$$
\begin{aligned}
H^{r}\left(\mathbb{T}^{3}\right)=\left\{\omega(x)=\sum_{k \in \mathbb{Z}^{3}} \hat{\omega}_{k} e^{i k \cdot x}: \hat{\omega}_{0}=0, \overline{\hat{\omega}_{k}}=\hat{\omega}_{-k},\right. \\
\left.\|\omega\|_{H^{r}}^{2}=(2 \pi)^{3} \sum_{k \in \mathbb{Z}^{3}}\left(1+|k|^{2}\right)^{r}\left|\hat{\omega}_{k}\right|^{2}<\infty\right\}
\end{aligned}
$$

is the periodic Sobolev space. For $r, \tau \geq 0$ define the normed spaces $Y_{r, \tau} \subset X_{r, \tau}$ by

$$
X_{r, \tau}=\bigcap_{m=1}^{3} D\left(\Lambda_{m}^{r} e^{\tau \Lambda_{m}}\right), \quad\|\omega\|_{X_{r, \tau}}^{2}=\sum_{m=1}^{3}\left\|\Lambda_{m}^{r} e^{\tau \Lambda_{m}} \omega\right\|_{L^{2}}^{2},
$$

and $Y_{r, \tau}=X_{r+1 / 2, \tau}$. In the following lemma we prove that the above defined spaces consist of real-analytic functions.

Lemma 2.4. If $\omega \in X_{r, \tau}$ for $r \geq 0$ and $\tau>0$, then $\omega$ is of Gevrey-class 1 (i.e., analytic), with uniform space analyticity radius at least $\tau / 3$.

Proof. It is sufficient to show that $\sum_{k \in \mathbb{Z}^{3}} e^{2 \tau|k| / 3}\left|\hat{\omega}_{k}\right|^{2}<\infty$ (cf. [K1, LO]). This follows from $\sum_{k \in \mathbb{Z}^{3}} e^{2 \tau|k| / 3}\left|\hat{\omega}_{k}\right|^{2} \leq\|\omega\|_{X_{r, \tau}}^{2}$, a direct consequence of the triangle inequality and the mean-zero condition.

Similarly, one can show that $X_{r, \tau}$ is equivalent to the subspace $\mathcal{D}\left((\sqrt{-\Delta})^{r} e^{\tau \sqrt{-\Delta}}\right)$ of Gevrey-class 1 which was used in $[\mathrm{LO}$. The following lemma is needed to prove Theorem 2.1.

Lemma 2.5. Let $m=1,2,3$ and $\omega \in Y_{r, \tau}$, where $r>7 / 2$. If $u=K * \omega$, where $K$ is the periodic Biot-Savart kernel, then

$$
\begin{aligned}
& \left|\left(u \cdot \nabla \omega, \Lambda_{m}^{2 r} e^{2 \tau \Lambda_{m}} \omega\right)\right|+\left|\left(\omega \cdot \nabla u, \Lambda_{m}^{2 r} e^{2 \tau \Lambda_{m}} \omega\right)\right| \\
& \leq C \\
& \quad\left(\tau\|\nabla u\|_{L^{\infty}}+\tau^{2}\|\omega\|_{H^{r}}+\tau^{2}\|\omega\|_{X_{r, \tau}}\right)\|\omega\|_{Y_{r, \tau}}\left\|\Lambda_{m}^{r+1 / 2} e^{\tau \Lambda_{m}} \omega\right\|_{L^{2}} \\
& \quad+C\left(\|\nabla u\|_{L^{\infty}}\|\omega\|_{X_{r, \tau}}+(1+\tau)\|\omega\|_{H^{r}}^{2}\right)\left\|\Lambda_{m}^{r} e^{\tau \Lambda_{m}} \omega\right\|_{L^{2}},
\end{aligned}
$$

where the positive constant $C$ depends only on $r$.

We note that Lemma 2.5 is an improvement of Lemma 8 in $\mathrm{LO}$. In the first term on the right of (2.2), the lowest power of $\tau$ is paired with the better behaved quantity $\|\nabla u\|_{L^{\infty}}$, while $\|\omega\|_{H^{r}}$ is paired with $\tau^{2}$. This implies algebraic rather than exponential dependence of $\tau(t)$ on the $H^{r}$-norm of $\omega$.

We prove Theorem 2.1 by showing that if the initial datum is of Gevrey-class 1 , the solution remains in this class as long as it exists. In the following $C$ denotes a generic positive constant depending on $r$. 
Proof of Theorem 2.1. We note that if the initial datum $\omega_{0}$ is real-analytic with radius of analyticity at least $\lambda \tau(0)$, with $\lambda>1$, then $\omega_{0} \in H^{r}$ and $\left\|e^{\tau(0) \sqrt{-\Delta}} \omega_{0}\right\|_{H^{r}}<$ $\infty$ (cf. [K1, LO]). Therefore $\omega_{0} \in X_{r, \tau(0)}$. We now prove that for all $0 \leq t<T$ the $H^{r}$-solution of (1.1)-(1.3) satisfies $\omega(\cdot, t) \in X_{r, \tau(t)}$, for an appropriate function $\tau(t)$. When no ambiguity arises, we suppress the time dependence of $\tau$ and $\omega$ on $t$.

By taking the $L^{2}$-inner product of (1.1) with $\Lambda_{m}^{2 r} e^{2 \tau \Lambda_{m}} \omega$, where $m=1,2,3$, we obtain

$$
\begin{aligned}
\frac{1}{2} \frac{d}{d t}\left\|\Lambda_{m}^{r} e^{\tau \Lambda_{m}} \omega\right\|_{L^{2}}^{2}= & \dot{\tau} \\
& \left\|\Lambda_{m}^{r+1 / 2} e^{\tau \Lambda_{m}} \omega\right\|_{L^{2}}^{2} \\
& -\left(u \cdot \nabla \omega, \Lambda_{m}^{2 r} e^{2 \tau \Lambda_{m}} \omega\right)+\left(\omega \cdot \nabla u, \Lambda_{m}^{2 r} e^{2 \tau \Lambda_{m}} \omega\right) .
\end{aligned}
$$

The constant $C$ in Lemma 2.5 can be taken large enough so that $\|\omega(\cdot, t)\|_{H^{r}}^{2} \leq$ $\left\|\omega_{0}\right\|_{H^{r}}^{2} g(t)$ for all $0 \leq t<T$, where $g(t)=\exp \left(C \int_{0}^{t}\|\nabla u(\cdot, s)\|_{L^{\infty}} d s\right)$. In order to conclude the proof, we sum over $m=1,2,3$ in (2.3) and use the estimate (2.2). We obtain

$$
\begin{aligned}
\frac{1}{2} \frac{d}{d t}\|\omega\|_{X_{r, \tau}}^{2} \leq C & \left(\|\nabla u\|_{L^{\infty}}\|\omega\|_{X_{r, \tau}}+(1+\tau)\|\omega\|_{H^{r}}^{2}\right)\|\omega\|_{X_{r, \tau}} \\
& +\left(\dot{\tau}+C \tau\|\nabla u\|_{L^{\infty}}+C \tau^{2}\|\omega\|_{H^{r}}+C \tau^{2}\|\omega\|_{X_{r, \tau}}\right)\|\omega\|_{Y_{r, \tau}}^{2} .
\end{aligned}
$$

If $\tau$ is such that the second term on the right of the above is negative, then $\tau$ is decreasing and

$$
\frac{d}{d t}\|\omega\|_{X_{r, \tau}} \leq C\|\nabla u\|_{L^{\infty}}\|\omega\|_{X_{r, \tau}}+C(1+\tau(0))\|\omega\|_{H^{r}}^{2} .
$$

By Gronwall's inequality this implies

$$
\|\omega(\cdot, t)\|_{X_{r, \tau(t)}} \leq g(t)\left(\left\|\omega_{0}\right\|_{X_{r, \tau(0)}}+C(1+\tau(0)) \int_{0}^{t}\|\omega(\cdot, s)\|_{H^{r}}^{2} g(s)^{-1} d s\right)=A(t) .
$$

A sufficient condition for the above to hold is that

$$
\dot{\tau}+C \tau\|\nabla u\|_{L^{\infty}}+C \tau^{2}\|\omega\|_{H^{r}}+C \tau^{2} A(t) \leq 0,
$$

for all $t \geq 0$. It suffices to set

$$
\tau(t)=g(t)^{-1}\left(\tau(0)^{-1}+C \int_{0}^{t}\left(\|\omega(\cdot, s)\|_{H^{r}}+A(s)\right) g(s)^{-1} d s\right)^{-1} .
$$

In particular, since $\|\omega(\cdot, t)\|_{H^{r}}^{2} \leq\left\|\omega_{0}\right\|_{H^{r}}^{2} g(t)$, we obtain

$$
\tau(t) \geq g(t)^{-1}\left(C^{\prime}+C^{\prime \prime} t^{2}\right)^{-1},
$$

where $C^{\prime}=2 / \tau(0)$ and the constant $C^{\prime \prime}$ depends on $r, \tau(0),\left\|\omega_{0}\right\|_{H^{r}}$, and $\left\|\omega_{0}\right\|_{X_{r, \tau(0)}}$.

\section{Proof of MAIN LEMma}

Before we start the proof of Lemma 2.5 we introduce the operators

$$
\Lambda f(x)=\sum_{k \in \mathbb{Z}^{3}}|k|_{1} \hat{f}_{k} e^{i x \cdot k}
$$


and

$$
H_{m} f(x)=\sum_{k \in \mathbb{Z}^{3}} \operatorname{sgn}\left(k_{m}\right) \hat{f}_{k} e^{i x \cdot k}, \quad m=1,2,3,
$$

for all $f \in H^{1}\left(\mathbb{T}^{3}\right)$. Here $|k|_{1}$ denotes $\left|k_{1}\right|+\left|k_{2}\right|+\left|k_{3}\right|$. The following $L^{2}$-estimates follow directly from Plancherel's theorem and the proofs are thus omitted.

Lemma 3.1. Let $\omega \in X_{r, \tau}$, for $\tau \geq 0$ and $r \geq 1$. Then for $m=1,2,3$ we have

$$
\left\|\Lambda_{m}^{r} \omega\right\|_{L^{2}} \leq\left\|\Lambda \Lambda_{m}^{r-1} \omega\right\|_{L^{2}} \leq C\|\omega\|_{H^{r}}
$$

and

$$
\left\|\nabla H_{m} \Lambda_{m}^{r-1} e^{\tau \Lambda_{m}} \omega\right\|_{L^{2}} \leq\left\|\Lambda \Lambda_{m}^{r-1} e^{\tau \Lambda_{m}} \omega\right\|_{L^{2}} \leq C\|\omega\|_{X_{r, \tau}} .
$$

Since $u=K * \omega$, an immediate consequence of the above is that $\left\|\Lambda_{m}^{r+1} u\right\|_{L^{2}} \leq$ $\left\|\Lambda \Lambda_{m}^{r} u\right\|_{L^{2}} \leq C\|\omega\|_{H^{r}}$, for a positive constant $C$.

Proof of Lemma 2.5. Let $m \in\{1,2,3\}$. In order to estimate $\left|\left(u \cdot \nabla \omega, \Lambda_{m}^{2 r} e^{2 \tau \Lambda_{m}} \omega\right)\right|$, we appeal to the cancellation property $\left(u \cdot \nabla \Lambda_{m}^{r} e^{\tau \Lambda_{m}} \omega, \Lambda_{m}^{r} e^{\tau \Lambda_{m}} \omega\right)=0$. Using Plancherel's theorem we obtain

$$
\begin{aligned}
(u \cdot \nabla \omega & \left., \Lambda_{m}^{2 r} e^{2 \tau \Lambda_{m}} \omega\right)=\left(u \cdot \nabla \omega, \Lambda_{m}^{2 r} e^{2 \tau \Lambda_{m}} \omega\right)-\left(u \cdot \nabla \Lambda_{m}^{r} e^{\tau \Lambda_{m}} \omega, \Lambda_{m}^{r} e^{\tau \Lambda_{m}} \omega\right) \\
= & i(2 \pi)^{3} \sum_{j+k+l=0}\left(\left|l_{m}\right|^{r} e^{\tau\left|l_{m}\right|}-\left|k_{m}\right|^{r} e^{\tau\left|k_{m}\right|}\right) \hat{u}_{j} \cdot k \hat{\omega}_{k}\left|l_{m}\right|^{r} e^{\tau\left|l_{m}\right|} \hat{\omega}_{l} \\
= & i(2 \pi)^{3} \sum_{j+k+l=0}\left(\left|l_{m}\right|^{r}-\left|k_{m}\right|^{r}\right) e^{\tau\left|k_{m}\right|} \hat{u}_{j} \cdot k \hat{\omega}_{k}\left|l_{m}\right|^{r} e^{\tau\left|l_{m}\right|} \hat{\omega}_{l} \\
& +i(2 \pi)^{3} \sum_{j+k+l=0}\left(e^{\tau\left|l_{m}\right|}-e^{\tau\left|k_{m}\right|}\right)\left|l_{m}\right|^{r} \hat{u}_{j} \cdot k \hat{\omega}_{k}\left|l_{m}\right|^{r} e^{\tau\left|l_{m}\right|} \hat{\omega}_{l}=T_{1}+T_{2},
\end{aligned}
$$

with $j, k, l \in \mathbb{Z}^{3}$. Recall that $\hat{\omega}_{0}=\hat{u}_{0}=0$. The first term on the far right side of the above is rewritten using the mean value theorem as

$$
\begin{gathered}
T_{1}=\operatorname{ir}(2 \pi)^{3} \sum_{j+k+l=0}\left(\left|l_{m}\right|-\left|k_{m}\right|\right)\left(\left(\theta_{m, k, l}\left|l_{m}\right|+\left(1-\theta_{m, k, l}\right)\left|k_{m}\right|\right)^{r-1}-\left|k_{m}\right|^{r-1}\right) \\
\times e^{\tau\left|k_{m}\right|} \hat{u}_{j} \cdot k \hat{\omega}_{k}\left|l_{m}\right|^{r} e^{\tau\left|l_{m}\right|} \hat{\omega}_{l} \\
\quad+i r(2 \pi)^{3} \sum_{j+k+l=0}\left(\left|l_{m}\right|-\left|k_{m}\right|\right)\left|k_{m}\right|^{r-1} e^{\tau\left|k_{m}\right|} \hat{u}_{j} \cdot k \hat{\omega}_{k}\left|l_{m}\right|^{r} e^{\tau\left|l_{m}\right|} \hat{\omega}_{l},
\end{gathered}
$$

for some $\theta_{m, k, l} \in(0,1)$. Since $j+k+l=0$, we have

$$
\begin{aligned}
\left|\left(\left|l_{m}\right|-\left|k_{m}\right|\right)\left(\left(\theta_{m, k, l}\left|l_{m}\right|+\left(1-\theta_{m, k, l}\right)\left|k_{m}\right|\right)^{r-1}-\left|k_{m}\right|^{r-1}\right)\right| \\
\leq C\left|j_{m}\right|^{2}\left(\left|j_{m}\right|^{r-2}+\left|k_{m}\right|^{r-2}\right) .
\end{aligned}
$$

The exponential factor is bounded as $e^{\tau\left|k_{m}\right|} \leq e+\tau^{2}\left|k_{m}\right|^{2} e^{\tau\left|k_{m}\right|}$, and $\left|\hat{u}_{j} \cdot k\right| \leq$ $C\left|\hat{u}_{j}\right||k|_{1}$, for a positive constant $C$. To estimate the second term on the right of (3.2) we use the decomposition

$$
\left|j_{m}+k_{m}\right|-\left|k_{m}\right|=j_{m} \operatorname{sgn}\left(k_{m}\right)+2\left(j_{m}+k_{m}\right) \operatorname{sgn}\left(j_{m}\right) \chi_{\left\{\operatorname{sgn}\left(k_{m}+j_{m}\right) \operatorname{sgn}\left(k_{m}\right)=-1\right\}} .
$$

(A version of the latter identity was also used by Lemarié-Rieusset in [L1, L2] for proving $L^{p}$-analyticity of solutions to the Navier-Stokes equations.) The first term 
in the decomposition (3.4) is treated using the Fourier inversion theorem. On the region $\left\{\operatorname{sgn}\left(k_{m}+j_{m}\right) \operatorname{sgn}\left(k_{m}\right)=-1\right\}$ we have $0 \leq\left|k_{m}\right| \leq\left|j_{m}\right|$ and thus in this region ||$l_{m}|-| k_{m}||\left|k_{m}\right|^{r-1} \leq C\left|j_{m}\right|^{r}$. We have thus proven

$$
\begin{gathered}
\left|T_{1}\right| \leq C \sum_{j+k+l=0}\left(\left|j_{m}\right|^{r}+\left|j_{m}\right|^{2}\left|k_{m}\right|^{r-2}\right)\left(e+\tau^{2}\left|k_{m}\right|^{2} e^{\tau\left|k_{m}\right|}\right)\left|\hat{u}_{j}\right||k|_{1}\left|\hat{\omega}_{k}\right|\left|l_{m}\right|^{r} e^{\tau\left|l_{m}\right|}\left|\hat{\omega}_{l}\right| \\
+C\left|\left(\partial_{m} u \cdot \nabla H_{m} \Lambda_{m}^{r-1} e^{\tau \Lambda_{m}} \omega, \Lambda_{m}^{r} e^{\tau \Lambda_{m}} \omega\right)\right| \\
\leq C\left(\|\omega\|_{H^{r}}^{2}+\|\nabla u\|_{L^{\infty}}\|\omega\|_{X_{r, \tau}}\right)\left\|\Lambda_{m}^{r} e^{\tau \Lambda_{m}} \omega\right\|_{L^{2}} \\
+C \tau^{2}\|\omega\|_{H^{r}}\|\omega\|_{Y_{r, \tau}}\left\|\Lambda_{m}^{r+1 / 2} e^{\tau \Lambda_{m}} \omega\right\|_{L^{2}} .
\end{gathered}
$$

In the second inequality we have appealed to the estimates in Lemma $3.1 r>7 / 2$, and $\left|k_{m}\right|^{1 / 2} \leq\left|j_{m}\right|^{1 / 2}+\left|l_{m}\right|^{1 / 2}$. Returning to (3.1) we write $T_{2}$ as

$$
\begin{aligned}
T_{2}= & i(2 \pi)^{3} \sum_{j+k+l=0}\left(e^{\tau\left(\left|l_{m}\right|-\left|k_{m}\right|\right)}-1-\tau\left(\left|l_{m}\right|-\left|k_{m}\right|\right)\right)\left|l_{m}\right|^{r-1 / 2} e^{\tau\left|k_{m}\right|} \\
& \times \hat{u}_{j} \cdot k \hat{\omega}_{k}\left|l_{m}\right|^{r+1 / 2} e^{\tau\left|l_{m}\right|} \hat{\omega}_{l} \\
+ & +i(2 \pi)^{3} \sum_{j+k+l=0} \tau\left(\left|l_{m}\right|-\left|k_{m}\right|\right)\left|k_{m}\right|^{r-1 / 2} e^{\tau\left|k_{m}\right|} \hat{u}_{j} \cdot k \hat{\omega}_{k}\left|l_{m}\right|^{r+1 / 2} e^{\tau\left|l_{m}\right|} \hat{\omega}_{l} \\
+ & +i(2 \pi)^{3} \sum_{j+k+l=0} \tau\left(\left|l_{m}\right|-\left|k_{m}\right|\right)\left(\left|l_{m}\right|^{r-1 / 2}-\left|k_{m}\right|^{r-1 / 2}\right) e^{\tau\left|k_{m}\right|} \\
& \times \hat{u}_{j} \cdot k \hat{\omega}_{k}\left|l_{m}\right|^{r+1 / 2} e^{\tau\left|l_{m}\right|} \hat{\omega}_{l} .
\end{aligned}
$$

The three terms on the right are treated as follows. Since $\left|e^{x}-1-x\right| \leq x^{2} e^{|x|}$, for all $x \in \mathbb{R}$, and $\left|l_{m}\right|^{r-1 / 2} \leq C\left(\left|j_{m}\right|^{r-1 / 2}+\left|k_{m}\right|^{r-1 / 2}\right)$, we obtain that the first term is bounded by

$$
C \tau^{2}\|\omega\|_{X_{r, \tau}}\|\omega\|_{Y_{r, \tau}}\left\|\Lambda_{m}^{r+1 / 2} e^{\tau \Lambda_{m}} \omega\right\|_{L^{2}} .
$$

The second term in the definition of $T_{2}$ above is treated using the decomposition (3.4). Note that in the region $\left\{\operatorname{sgn}\left(k_{m}+j_{m}\right) \operatorname{sgn}\left(k_{m}\right)=-1\right\}$ we have $0 \leq\left|k_{m}\right| \leq\left|j_{m}\right|$ and $0 \leq\left|l_{m}\right| \leq 2\left|j_{m}\right|$, and hence $e^{\tau\left|k_{m}\right|} \leq 1+\tau\left|j_{m}\right| e^{\tau\left|j_{m}\right|}$. Therefore, the second term in (3.6) is bounded by

$$
\begin{aligned}
& C \tau \sum_{j+k+l=0}\left|j_{m}\right|^{r+1 / 2}\left(1+\tau\left|j_{m}\right| e^{\tau\left|j_{m}\right|}\right)\left|\hat{u}_{j}\right||k|_{1}\left|\hat{\omega}_{k}\right|\left|l_{m}\right|^{r+1 / 2} e^{\tau\left|l_{m}\right|}\left|\hat{\omega}_{l}\right| \\
& \quad+C \tau\left|\left(\partial_{m} u \cdot \nabla H_{m} \Lambda_{m}^{r-1 / 2} e^{\tau \Lambda_{m}} \omega, \Lambda_{m}^{r+1 / 2} e^{\tau \Lambda_{m}} \omega\right)\right| \\
& \leq C \tau\|\omega\|_{H^{r}}^{2}\left\|\Lambda_{m}^{r} e^{\tau \Lambda_{m}} \omega\right\|_{L^{2}} \\
& \quad+C\left(\tau\|\nabla u\|_{L^{\infty}}+\tau^{2}\|\omega\|_{H^{r}}\right)\|\omega\|_{Y_{r, \tau}}\left\|\Lambda_{m}^{r+1 / 2} e^{\tau \Lambda_{m}} \omega\right\|_{L^{2}} .
\end{aligned}
$$

Using $e^{\tau\left|k_{m}\right|} \leq 1+\tau\left|k_{m}\right| e^{\tau\left|k_{m}\right|}$, the mean value theorem, and the triangle inequality, we obtain that the third term on the right side of (3.6) is bounded by

$$
C \tau\|\omega\|_{H^{r}}^{2}\left\|\Lambda_{m}^{r} e^{\tau \Lambda_{m}} \omega\right\|_{L^{2}}+C \tau^{2}\|\omega\|_{H^{r}}\|\omega\|_{Y_{r, \tau}}\left\|\Lambda_{m}^{r+1 / 2} e^{\tau \Lambda_{m}} \omega\right\|_{L^{2}} .
$$

Collecting (3.7)-(3.9) and the estimate on $T_{1}$ obtained earlier, we have proven that the term $\left|\left(u \cdot \nabla \omega, \Lambda_{m}^{2 r} e^{2 \tau \Lambda_{m}} \omega\right)\right|$ is bounded by the right of (2.2). 
The vorticity stretching term $\left(\omega \cdot \nabla u, \Lambda_{m}^{2 r} e^{2 \tau \Lambda_{m}} \omega\right)$ is treated similarly. We do not use the cancelation property, but instead subtract $\left(\omega \cdot \nabla \Lambda_{m}^{r} e^{\tau \Lambda_{m}} u, \Lambda_{m}^{r} e^{\tau \Lambda_{m}} \omega\right)+$ $\left(\Lambda_{m}^{r} e^{\tau \Lambda_{m}} \omega \cdot \nabla u, \Lambda_{m}^{r} e^{\tau \Lambda_{m}} \omega\right)$. By Hölder's inequality and Lemma 3.1 we have

$$
\begin{aligned}
\left|\left(\omega \cdot \nabla \Lambda_{m}^{r} e^{\tau \Lambda_{m}} u, \Lambda_{m}^{r} e^{\tau \Lambda_{m}} \omega\right)\right|+\left|\left(\Lambda_{m}^{r} e^{\tau \Lambda_{m}} \omega \cdot \nabla u, \Lambda_{m}^{r} e^{\tau \Lambda_{m}} \omega\right)\right| \\
\quad \leq C\|\nabla u\|_{L^{\infty}}\|\omega\|_{X_{r, \tau}}\left\|\Lambda_{m}^{r} e^{\tau \Lambda_{m}} \omega\right\|_{L^{2}},
\end{aligned}
$$

for a positive constant $C$ depending only on $r$. Thus in order to estimate the term $\left(\omega \cdot \nabla u, \Lambda_{m}^{2 r} e^{2 \tau \Lambda_{m}} \omega\right)$ it is sufficient to bound

$\left(\omega \cdot \nabla u, \Lambda_{m}^{2 r} e^{2 \tau \Lambda_{m}} \omega\right)-\left(\omega \cdot \nabla \Lambda_{m}^{r} e^{\tau \Lambda_{m}} u, \Lambda_{m}^{r} e^{\tau \Lambda_{m}} \omega\right)-\left(\Lambda_{m}^{r} e^{\tau \Lambda_{m}} \omega \cdot \nabla u, \Lambda_{m}^{r} e^{\tau \Lambda_{m}} \omega\right)$

$$
=i(2 \pi)^{3} \sum_{j+k+l=0}\left(\left|l_{m}\right|^{r} e^{\tau\left|l_{m}\right|}-\left|k_{m}\right|^{r} e^{\tau\left|k_{m}\right|}-\left|j_{m}\right|^{r} e^{\tau\left|j_{m}\right|}\right) \hat{\omega}_{j} \cdot k \hat{u}_{k}\left|l_{m}\right|^{r} e^{\tau\left|l_{m}\right|} \hat{\omega}_{l},
$$

where $j, k, l \in \mathbb{Z}^{3}$. We rewrite the left side of (3.10) as

$$
\begin{aligned}
& i(2 \pi)^{3} \sum_{j+k+l=0}\left(\left|l_{m}\right|^{r}-\left|j_{m}\right|^{r}\right)\left(e^{\tau\left|l_{m}\right|}-e^{\tau\left|k_{m}\right|}\right) \hat{\omega}_{j} \cdot k \hat{u}_{k}\left|l_{m}\right|^{r} e^{\tau\left|l_{m}\right|} \hat{\omega}_{l} \\
& +i(2 \pi)^{3} \sum_{j+k+l=0}\left(\left|l_{m}\right|^{r}-\left|k_{m}\right|^{r}-\left|j_{m}\right|^{r}\right) e^{\tau\left|k_{m}\right|} \hat{\omega}_{j} \cdot k \hat{u}_{k}\left|l_{m}\right|^{r} e^{\tau\left|l_{m}\right|} \hat{\omega}_{l} \\
& \quad+i(2 \pi)^{3} \sum_{j+k+l=0}\left|j_{m}\right|^{r}\left(e^{\tau\left|l_{m}\right|}-e^{\tau\left|j_{m}\right|}\right) \hat{\omega}_{j} \cdot k \hat{u}_{k}\left|l_{m}\right|^{r} e^{\tau\left|l_{m}\right|} \hat{\omega}_{l}=\widetilde{T}_{1}+\widetilde{T}_{2}+\widetilde{T}_{3} .
\end{aligned}
$$

The above terms are estimated in absolute value as follows. The mean value theorem and $e^{x} \leq 1+x e^{x}$, for $x \geq 0$, imply

$$
\left|\left(\left|l_{m}\right|^{r}-\left|j_{m}\right|^{r}\right)\left(e^{\tau\left|l_{m}\right|}-e^{\tau\left|k_{m}\right|}\right)\right| \leq C \tau\left(\left|k_{m}\right|^{r}\left|j_{m}\right|+\left|k_{m}\right|\left|j_{m}\right|^{r}\right) e^{\tau\left|j_{m}\right|} e^{\tau\left|k_{m}\right|} .
$$

Combined with $e^{x} \leq 1+x e^{x}$, for all $x \geq 0$, and the triangle inequality, (3.11) gives

$$
\begin{aligned}
& \left|\widetilde{T}_{1}\right| \leq C \tau\|\omega\|_{H^{r}}^{2}\left\|\Lambda_{m}^{r} e^{\tau \Lambda_{m}} \omega\right\|_{L^{2}} \\
& \quad+C \tau^{2}\left(\|\omega\|_{H^{r}}+\|\omega\|_{X_{r, \tau}}\right)\|\omega\|_{Y_{r, \tau}}\left\|\Lambda_{m}^{r+1 / 2} e^{\tau \Lambda_{m}} \omega\right\|_{L^{2}} .
\end{aligned}
$$

Similarly, by the mean value theorem we have

$$
\left.\left.\left|\left(\left|l_{m}\right|^{r}-\left|k_{m}\right|^{r}\right)-\right| j_{m}\right|^{r}|\leq C| j_{m}\left|\left(\left|j_{m}\right|^{r-1}+\left|k_{m}\right|^{r-1}\right)+\right| j_{m}\right|^{r} .
$$

Since $e^{x} \leq e+x^{2} e^{x}$, for all $x \geq 0$, the above implies

$$
\left|\widetilde{T}_{2}\right| \leq C\|\omega\|_{H^{r}}^{2}\left\|\Lambda_{m}^{r} e^{\tau \Lambda_{m}} \omega\right\|_{L^{2}}+C \tau^{2}\|\omega\|_{H^{r}}\|\omega\|_{Y_{r, \tau}}\left\|\Lambda_{m}^{r+1 / 2} e^{\tau \Lambda_{m}} \omega\right\|_{L^{2}} .
$$

The third term $\widetilde{T}_{3}$ is bounded similarly to the first one, but instead of (3.11) we use the estimate $\left|e^{\tau\left|l_{m}\right|}-e^{\tau\left|j_{m}\right|}\right| \leq C \tau\left|k_{m}\right| e^{\tau\left|j_{m}\right|} e^{\tau\left|k_{m}\right|}$ and obtain

$$
\begin{aligned}
& \left|\widetilde{T}_{3}\right| \leq C \tau\|\omega\|_{H^{r}}^{2}\left\|\Lambda_{m}^{r} e^{\tau \Lambda_{m}} \omega\right\|_{L^{2}} \\
& +C \tau^{2}\left(\|\omega\|_{H^{r}}+\|\omega\|_{X_{r, \tau}}\right)\|\omega\|_{Y_{r, \tau}}\left\|\Lambda_{m}^{r+1 / 2} e^{\tau \Lambda_{m}} \omega\right\|_{L^{2}} .
\end{aligned}
$$

This concludes the proof of the lemma.

Remark 3.2. By working in $X_{r, \tau, s}=\bigcap_{m=1}^{3} \mathcal{D}\left(\Lambda_{m}^{r} e^{\tau \Lambda_{m}^{s}}\right)$, for $s \in(0,1)$, one can show that the radius of Gevrey-class $s$ regularity (cf. [FT, LO] for a definition of the Gevrey classes) of the smooth solution to (1.1) $-(1.3)$ satisfies the same lower bound 
(2.1) as in Theorem 2.1, given that the initial datum is of Gevrey-class $s$. As in Foias and Temam [FT] the proof carries over directly from the analytic case $s=1$ and relies on the fact that for $s \leq 1$ we have $|k+j|^{s} \leq|k|^{s}+|j|^{s}$, and for $s<1$ we additionally use

$$
\left.|| l_{m}\right|^{s}-\left|k_{m}\right|^{s}|\leq C|\left|l_{m}\right|-\left|k_{m}\right| \mid \frac{1}{\left|l_{m}\right|^{1-s}+\left|k_{m}\right|^{1-s}}
$$

where $C$ is a positive constant depending only on $s$. The latter inequality is for instance used to estimate the term $T_{2}$ defined in (3.6). Identity (3.4) still needs to be used in the Gevrey case.

\section{REFERENCES}

AM. S. Alinhac and G. Metivier, Propagation of local analyticity for the Euler equation, Proceedings of Symposia in Pure Mathematics, Vol. 43, pp. 1-4, AMS, Providence, Rhode Island, 1985. MR812279

B. C. Bardos, Analyticité de la solution de l'équation d'Euler dans un ouvert de $\mathbb{R}^{n}$, C. R. Acad. Sci. Paris Sér. A-B 283 (1976), A255-A258. MR0425393 (54:13349)

BB. C. Bardos and S. Benachour, Domaine d'analyticité des solutions de l'équation d'Euler dans un ouvert de $\mathbb{R}^{n}$, Ann. Scuola Norm. Sup. Pisa Cl. Sci. (4) 4 (1977), 647-687. MR.0454413 (56:12664)

BBZ. C. Bardos, S. Benachour, and M. Zerner, Analyticité des solutions périodiques de l'équation d'Euler en deux dimensions, C. R. Acad. Sci. Paris Sér. A-B 282 (1976), A995-A998. MR0410094 (53:13844)

Be. $\quad$ S. Benachour, Analyticité des solutions périodiques de l'équation d'Euler en trois dimensions, C. R. Acad. Sci. Paris Sér. A-B 283 (1976), A107-A110. MR0425323 (54:13279)

BG. M. S. Baouendi and C. Goulaouic, Problèmes de Cauchy pseudo-différentiels analytiques, Séminaires Goulaouic-Schwarz, 1975-1976, École Polytechnique, Asterisque, No. 34-35, Soc. Math. France, 1976. MR0481385 (58:1502)

BGK1. J.L. Bona, Z. Grujić, and H. Kalisch, Algebraic lower bounds for the uniform radius of spatial analyticity for the generalized KdV equation, Ann. Inst. H. Poincaré Anal. Non Linéaire 22 (2005), no. 6, 783-797. MR2172859 (2006e:35282)

BGK2. J.L. Bona, Z. Grujić, and H. Kalisch, Global solutions of the derivative Schrödinger equation in a class of functions analytic in a strip, J. Differential Equations 229 (2006), no. 1, 186-203. MR2265624 (2007k:35453)

BKM. J.T. Beale, T. Kato, and A. Majda, Remarks on the breakdown of smooth solutions for the 3-D Euler equations, Comm. Math. Phys. 94 (1984), no. 1, 61-66. MR763762 (85j:35154)

CTV. P. Constantin, E.S. Titi, and J. Vukadinović, Dissipativity and Gevrey regularity of a Smoluchowski equation, Indiana Univ. Math. J. 54 (2005), no. 4, 949-969. MR2164412 (2006e:35162)

EB. D.G. Ebin and J.E. Marsden, Groups of diffeomorphisms and the solution of the classical Euler equations for a perfect fluid, Bull. Amer. Math. Soc. 75 (1969), 962-967. MR0246328 (39:7632)

FT. C. Foias and R. Temam, Gevrey class regularity for the solutions of the Navier-Stokes equations, J. Funct. Anal. 87 (1989), 359-369. MR1026858(91a:35135)

FTi. A.B. Ferrari and E.S. Titi, Gevrey regularity for nonlinear analytic parabolic equations, Comm. Partial Differential Equations 23 (1998), no. 1-2, 1-16. MR1608488 (99a:35116)

GK1. Z. Grujić and I. Kukavica, Space analyticity for the nonlinear heat equation in a bounded domain, J. Differential Equations 154 (1999), no. 1, 42-54. MR.1685650 (2000a:35104)

GK2. Z. Grujić and I. Kukavica, Space analyticity for the Navier-Stokes and related equations with initial data in $L^{p}$, J. Funct. Anal. 152 (1998), no. 2, 447-466. MR1607936 (99b:35168)

J. V.I. Judovič, Non-stationary flows of an ideal incompressible fluid, Zh. Vych. Mat. i Mat. Fiz. 3 (1963), 1032-1066. MR0158189 (28:1415)

K1. I. Kukavica, Hausdorff length of level sets for solutions of the Ginzburg-Landau equation, Nonlinearity 8 (1995), no. 2, 113-129. MR.1328590 (96a:35198) 
K2. I. Kukavica, On the dissipative scale for the Navier-Stokes equation, Indiana Univ. Math. J. 48 (1999), no. 3, 1057-1081. MR1736969 (2001i:76032)

Ka. T. Kato, Nonstationary flows of viscuous and ideal fluids in $\mathbb{R}^{3}$, J. Functional Analysis 9 (1972), 296-305. MR0481652 (58:1753)

L1. P.G. Lemarié-Rieusset, Une remarque sur l'analyticité des solutions milds des équations de Navier-Stokes dans $R^{3}$, C. R. Acad. Sci. Paris Sér. I Math. 330 (2000), no. 3, 183-186. MR.1748305 (2001c:35190)

L2. P.G. Lemarié-Rieusset, Nouvelles remarques sur l'analyticité des solutions milds des équations de Navier-Stokes dans $\mathbb{R}^{3}$, C. R. Math. Acad. Sci. Paris 338 (2004), no. 6, 443-446. MR2057722 (2005a:35222)

Lb. D. Le Bail, Analyticité locale pour les solutions de l'équation d'Euler, Arch. Rational Mech. Anal. 95 (1986), no. 2, 117-136. MR850093 (88i:35134)

LO. C.D. Levermore and M. Oliver, Analyticity of solutions for a generalized Euler equation, J. Differential Equations 133 (1997), no. 2, 321-339. MR.1427856 (97k:35198)

MB. A.J. Majda and A.L. Bertozzi, Vorticity and incompressible flow, Cambridge Texts in Applied Mathematics, vol. 27, Cambridge University Press, Cambridge, 2002. MR.1867882 (2003a:76002)

OT. M. Oliver and E.S. Titi, On the domain of analyticity of solutions of second order analytic nonlinear differential equations, J. Differential Equations 174 (2001), no. 1, 55-74. MR.1844523 (2002d:35007)

T. R. Temam, On the Euler equations of incompressible perfect fluids, J. Functional Analysis 20 (1975), no. 1, 32-43. MR0430568 (55:3573)

Department of Mathematics, University of Southern California, 3620 S. Vermont Avenue, Los Angeles, California 90089

E-mail address: kukavica@usc.edu

Department of Mathematics, University of Southern California, 3620 S. Vermont Avenue, Los Angeles, California 90089

E-mail address: vicol@usc.edu 